\title{
Socioeconomic Inequalities in the Prevalence and Management of Childhood Diarrhoea: Potential Health Gains to be Achieved
}

\author{
Birger C. Forsberg ${ }^{*}$, , Davidson Gwatkin ${ }^{\mathrm{b}}$, Göran Tomson ${ }^{\mathrm{a}}$, Peter Allebeck ${ }^{\mathrm{a}}$ and Max G. Petzold ${ }^{\mathrm{c}}$ \\ ${ }^{a}$ Department of Public Health Sciences, Karolinska Institutet, S-171 76 Stockholm, Sweden \\ ${ }^{b}$ Health Nutrition Population, World Bank, Washington, USA \\ ${ }^{c}$ Nordic School of Public Health, Gothenburg, Sweden
}

\begin{abstract}
Background: Diarrhoea represents a major burden of childhood disease in countries with low and middle incomes. The need for targeting poorer socioeconomic groups has been forwarded as a priority in diarrhoea control. The aim of this study was to determine the role of socioeconomic inequalities in the control of childhood diarrhoea.

Methods: Household data on prevalence and case management of diarrhoea in children aged 0-4 years from 93 Demographic Health Surveys (DHS) in 55 countries in 1990-2005 were analysed by wealth quintiles. Socioeconomic disparities and trends in inequalities were assessed.

Findings: Households in the poorest socioeconomic group consistently showed higher morbidity attributable to diarrhoea and less frequent use of oral rehydration therapy (ORT) when compared with households in the richest socioeconomic group. If the prevalence of diarrhoea in all households were the same as that in the best-performing wealth quintiles, the prevalence of diarrhoea would be reduced by $22.8 \%$. If all socioeconomic groups had adopted the ORT-use rates achieved by the best-performing wealth quintiles, overall use of ORT would have increased by $21.5 \%$. This corresponds to an increase in treated episodes of diarrhoea of 481 million annually in the study population. Trend analysis did not show significant changes in inequalities over time.

Interpretations: To increase efficiency in the control of diarrhoeal diseases, preventive measures should be targeted to the poor, rehydration products should be made more affordable and information on the importance of using ORT should be disseminated more intensively to disadvantaged groups.
\end{abstract}

Keywords: Diarrhoea, disease control, oral rehydration, inequalities, child health, health policy.

\section{BACKGROUND}

Diarrhoea represents a major burden of disease in lowand middle-income countries, where it is still a significant cause of death in childhood. The average number of episodes per child aged less than 5 years was estimated at 3.2 per year in a study reported in 2003 [1]. There has been little progress in reducing the incidence of diarrhoea over the past decade [2]. A global review of childhood mortality from 2003 suggested that about $22 \%$ of an estimated 10.8 million deaths in children aged less than 5 years were caused by diarrhoea [3].

An effective way to reduce mortality caused by diarrhoea is promotion and use of oral rehydration therapy (ORT), which was established as a large-scale public health intervention more than 25 years ago [4]. ORT is defined as the use of solutions based on oral rehydration salts (ORS) or recommended home fluids (RHF) [5]. ORS refers to a prepackaged powder containing at least glucose and sodium in such combinations that the resulting solution leads to active reabsorption of sodium and water [6]. Such solutions can be used for treatment of dehydration. RHF refers to household

*Address correspondence to this author at the Department of Public Health Sciences, Karolinska Institutet, S-171 76 Stockholm, Sweden;

E-mail: Birger.Forsberg@ki.se fluids that can be recommended for the prevention and/or treatment of dehydration, e.g. rice water, local teas or various types of soup [5].

Although the use of ORT increased significantly in the 1980s [7], recent data suggest that use has now reached a plateau at about $40 \%$ of children with diarrhoea [8]. In the same study [8] it was found that some 300 million children did not get ORT when they suffered from diarrhoea. Thus even now, at the beginning of the 21 st century, a large number of children with diarrhoea do not get appropriate treatment; this is a major concern for improving child health and attaining Millennium Development Goal 4 (MDG 4), the reduction of child mortality.

New approaches to ensure that all children suffering from diarrhoea are treated must be discussed and evaluated. The need for targeting the poor to improve the coverage of child health programmes has been forwarded as a priority in this context [9]. Inequities in the seeking of health care are a documented obstacle to further progress in the case management of diarrhoea [10] and in child survival [11].

The aims of this study were to estimate inequalities in morbidity caused by diarrhoea and in use of ORT in order to ascertain the potential for health improvements via the targeting of interventions to socioeconomically disadvantaged groups. 


\section{METHODS}

We analysed data on the prevalence and management of diarrhoea from surveys carried out by Demographic and Health Surveys (DHS). DHS surveys are surveys carried out in many countries since 1986 under the guidance of a common support programme and following standard procedures for questionnaire design, data collection and data analysis [12]. The surveys select a nationally representative sample typically consisting of 5000 to 30000 households. In the household, interviews on reproductive health, child health and nutrition are carried out with women aged 15-49 years. Within a country, DHS surveys are ideally conducted every 5 years. Use of a standardized core questionnaire in DHS surveys allows for comparisons across countries and over time.

For each survey, we sought information on two outcome indicators:

- Prevalence of diarrhoea, defined as percentage of children aged $0-4$ years with diarrhoea in the 2 weeks preceding the household interview;

- $\quad$ Use of ORT, defined as percentage of children aged $0-4$ years with diarrhoea in the 2 weeks preceding the household interview and that was treated with ORT (either ORS and/or RHF).

To classify socioeconomic status from the DHS data, we used a composite asset index based on principal component analysis (PCA). The index has been described in detail elsewhere [13]. Typical components of the index are materials used for house floors, walls and roofs, possession of items like fan, radio, television set, watch, bicycle or other means of transport, source of water and sanitary facilities. The asset index was calculated for each household as determined by application of PCA. The resulting household scores were standardized to have a mean of 0 and a standard deviation of 1.

Based on the asset index, we classified households into quintiles reflecting their level of wealth. Prevalence of diarrhoea and use of ORT were then calculated for each of the wealth quintiles and the results were compared.

Data on the prevalence of diarrhoea were taken from 93 DHS surveys conducted in 55 countries in 1990-2005. Use of ORT by quintile was available from 87 of those surveys. Population data were extracted from the International Data Base, which provides mid-year estimates of the population aged $0-4$ years for all countries and years used in the study [14].

With standard procedures for calculating diarrhoea incidence from prevalence based on the estimated average duration of a diarrhoea episode [15], the 2-week prevalence found in each of the surveys was converted into a 2-week incidence estimate. This estimate from each of the surveys was then used to calculate a weighted average for the annual number of episodes of diarrhoea per child in the total population studied. This estimate of 3.6 episodes per year was then used to calculate the annual absolute number of episodes of diarrhoea in the total population of children surveyed. With this estimate potential improvements, expressed as a reduction in the annual number of episodes of diarrhoea, could be determined.
The absolute number of children having received ORT was calculated by multiplying the number of children with diarrhoea by the ORT-use rate found in the survey.

In order to assess the number of potentially preventable cases of diarrhoea in children aged 0-4 years, we calculated the lowest potential prevalence of diarrhoea for the whole population surveyed by applying the lowest prevalence found in any of the quintiles to the whole study population. The potential reduction in prevalence of diarrhoea for the whole population was then given as a percentage of the actual prevalence. In a similar manner, we estimated the potential increase in use of ORT for the whole population by applying the highest rate of use of ORT in any quintile to the population with documented diarrhoea. The potential increase in use of ORT was then calculated as a percentage of the actual use of ORT.

For each country, the potential reduction in morbidity caused by diarrhoea and the potential increase in use of ORT were combined to calculate the best possible improvement in diarrhoea control that could potentially be achieved. In this situation, the prevalence of diarrhoea and use of ORT in all socioeconomic groups would be equal to that in the bestperforming quintiles, respectively, and all within-country inequalities would have been removed. The resulting potential improvement was expressed as reduction in the number of children that would not receive ORT when they had diarrhoea, these being the children that would be at the greatest risk of dying from dehydration.

Aggregated data by WHO region ${ }^{1}$ were calculated from available survey data from countries in the region.

Trends in mean rate ratios for prevalence of diarrhoea and use of ORT by wealth quintiles were estimated for all 32 countries in which more than one survey had been conducted during the study period. The prevalence ratio was defined as the prevalence rate (\%) in the highest wealth quintile divided by the prevalence rate (\%) in the lowest quintile. Similarly, the ORT-use ratio was defined as the rate of use of ORT (\%) in the highest wealth quintile divided by the rate of use of ORT (\%) in the lowest quintile. Also, trends in differences in rates between the highest and lowest wealth quintiles were determined. In this calculation, the variable used was the difference expressed as a proportion of the rate in the highest wealth quintile. In both instances, trends were assessed using a multi-level linear regression model with a random countryspecific intercept and a common slope.

\section{RESULTS}

\section{Prevalence of Diarrhoea}

A total of 635.1 million children were surveyed during the study period. Of these, $52 \%$ were in the WHO SouthEast Asia Region and $23 \%$ in the WHO African Region (Table 1).

For all surveys combined, the 2-week prevalence of diarrhoea in the lowest quintile was $16.3 \%$, while it was $11.6 \%$ in the highest quintile. The prevalence of diarrhoea for all households taken together was $14.7 \%$ (Table 2).

\footnotetext{
${ }^{1}$ For listings of the countries associated with the WHO regions, please see http://www.who.int/about/structure/en/index.html.
} 
Table 1. Number of DHS Surveys Studied, Number of Countries, Time Period and Number of Children Aged 0-4 Years Surveyed, by WHO Region

\begin{tabular}{|c|c|c|c|c|}
\hline WHO Region & Number of Surveys & Number of Countries & Period (Years) & Children Aged 0-4 Years Surveyed (Millions) \\
\hline \hline African & 47 & 29 & $1990-2004$ & 148.3 \\
\hline Americas & 17 & 9 & $1990-2005$ & 47.9 \\
\hline South-East Asia & 9 & 4 & $1990-2004$ & 19.9 \\
\hline European & 8 & 6 & $1993-2000$ & 47.9 \\
\hline Eastern Mediterranean & 7 & 4 & $1992-2004$ & 38.4 \\
\hline Western Pacific & 5 & 3 & $1997-2003$ & 635.1 \\
\hline
\end{tabular}

DHS: Demographic Health Survey.

Table 2. Prevalence of Diarrhoea and Use of ORT in the Wealthiest and Poorest Households (Highest and Lowest Quintiles), by WHO Region

\begin{tabular}{|c|c|c|c|c|c|c|}
\hline \multirow{2}{*}{ WHO Region } & \multicolumn{3}{|c|}{ Prevalence of Diarrhoea } & \multicolumn{3}{|c|}{ Use of ORT } \\
\hline & Poorest $^{\mathrm{a}}(\%)$ & Wealthiest $^{\mathrm{b}}(\%)$ & All (\%) & Poorest $^{\mathrm{a}}(\%)$ & Wealthiest $^{\mathrm{b}}(\%)$ & All (\%) \\
\hline African & 20.8 & 14.3 & 19.0 & 49.1 & 69.6 & 56.1 \\
\hline Americas & 18.9 & 9.6 & 15.4 & 61.1 & 68.9 & 66.0 \\
\hline South-East Asia & 13.9 & 11.0 & 13.1 & 41.7 & 57.8 & 46.9 \\
\hline European & 25.8 & 15.3 & 20.9 & 59.0 & 72.3 & 68.9 \\
\hline Eastern Mediterranean & 15.5 & 12.1 & 13.9 & 46.4 & 61.3 & 54.9 \\
\hline Western Pacific & 12.5 & 6.7 & 10.2 & 70.7 & 80.2 & 74.2 \\
\hline World & 16.3 & 11.6 & 14.7 & 47.7 & 63.7 & 53.5 \\
\hline
\end{tabular}

ORT: oral rehydration therapy.

${ }^{\mathrm{a}}$ The poorest $20 \%$ of households, i.e. the lowest wealth quintile.

${ }^{\mathrm{b}}$ The wealthiest $20 \%$ of households, i.e. the highest wealth quintile.

When comparing the prevalence of diarrhoea in the quintiles containing the wealthiest and the poorest households, respectively, prevalence was lower in the wealthiest quintile (the wealthiest $20 \%$ of households) in $88.2 \%$ of the surveys. Only in four surveys $(4.3 \%)$ was the lowest prevalence of diarrhoea of all quintiles reported to be in the poorest quintile (the poorest $20 \%$ of households).

The prevalence of diarrhoea would be reduced by $22.8 \%$ among the 635.1 million children surveyed if the prevalence in all socioeconomic groups could be reduced to that in the quintile with the lowest prevalence (Table 3).

Assuming that, on average, a child has 3.6 episodes of diarrhoea annually, a reduction of $22.8 \%$ corresponds to 511.2 million episodes of diarrhoea fewer per year among the 635.1 million children surveyed. The largest potential reductions in morbidity caused by diarrhoea were in the WHO Region of the Americas (37.6\%) and in the WHO Western Pacific Region (34.7\%).

If the prevalence of diarrhoea in the lowest wealth quintile were reduced to that in the highest wealth quintile, the prevalence of diarrhoea would be reduced by almost one third $(28.8 \%)$ in this group comprising the poorest of the population.

\section{Use of ORT}

A total of 629.8 million children were surveyed in the 87 surveys from which information on use of ORT was available by quintile. For all these surveys combined, use of ORT was $47.7 \%$ in the lowest quintile and $63.7 \%$ in the highest quintile. The average use of ORT for all surveys was $53.5 \%$ (Table 2).

Comparing the richest and the poorest by wealth quintile showed that use of ORT was more frequent in the highest quintile than in the lowest quintile in $89.7 \%$ of the surveys. The WHO Eastern Mediterranean Region had the lowest proportion of surveys $(71.4 \%)$ in which use of ORT was most common for the wealthiest.

The use of ORT would have increased by $21.5 \%$ if all socioeconomic groups had adopted the use pattern of the best-performing quintile (Table 3). Assuming that among the 629.8 million children surveyed each child had 3.6 episodes of diarrhoea per year, an increase in the use of ORT of $21.5 \%$ would correspond to an additional 480.6 million episodes of childhood diarrhoea per year being treated with ORT. The largest potential gains in use of ORT were found in the WHO African Region (25.1\%) and in the WHO South-East Asia Region (24.1\%). 
Table 3. Potential Health Gains to be Achieved: Reduction in the Prevalence of Diarrhoea, Increase in Use of ORT and Decrease in the Number of Children not Receiving ORT if All Households had had the Same Performance as the Best-Performing Quintile of Households, by WHO Region

\begin{tabular}{|c|c|c|c|}
\hline WHO region & $\begin{array}{c}\text { Reduction in Prevalence } \\
\text { of Diarrhoea (\%) }\end{array}$ & Increase in Use of ORT (\%) & $\begin{array}{c}\text { Decrease in Number of Children Not } \\
\text { Receiving ORT (\%) }\end{array}$ \\
\hline \hline African & 27.8 & 25.1 & 52.2 \\
\hline Americas & 37.6 & 13.7 & 53.8 \\
\hline South-East Asia & 15.9 & 24.1 & 33.1 \\
\hline European & 31.8 & 14.7 & 50.1 \\
\hline Eastern Mediterranean & 17.2 & 16.1 & 33.5 \\
\hline Western Pacific & 34.7 & 9.4 & 53.0 \\
\hline World & 22.8 & 21.5 & 40.8 \\
\hline
\end{tabular}

If the households in the lowest quintiles were to have the same use of ORT as those in the highest quintiles, ORT use would increase by $17.3 \%$. Such an increase would signify a relative improvement in use of ORT of $37.2 \%$ for those in the poorest quintile.

\section{Potential Health Gains from Combined Reductions in the Prevalence of Diarrhoea and Increase in Use of ORT}

Reduction in the prevalence of diarrhoea would mean that fewer children got diarrhoea and therefore fewer children would be in need of ORT. Increase in the use of ORT would mean that more children would get treated with ORT and in consequence fewer children would fail to receive ORT. Not receiving ORT increases the risk of developing dehydration, which can be fatal.

To assess the full consequences of socioeconomic inequalities, we estimated the potential reduction in the number of children not receiving ORT if the prevalence of diarrhoea and use of ORT among all the children in each survey were equal to that of the best-performing quintiles in that survey. The number of episodes of diarrhoea not treated with ORT would then be reduced by $40.8 \%$ among all the children surveyed (Table 3). The largest potential gains were in the WHO Region of the Americas (53.8\%), the WHO Western Pacific region (53.0\%) and the WHO African Region (52.2\%).

\section{Trend Analysis}

The analysis of data on trends in the countries where several surveys had been conducted showed a small tendency for disparities in prevalence of diarrhoea to decrease and for disparities in ORT use to increase, although none of the changes were statistically significant (Fig. 1). The same applied to trends in proportional differences between the highest and lowest wealth quintiles.

\section{DISCUSSION}

We found a consistent pattern of inequalities in the prevalence of morbidity caused by diarrhoea and in treatment with ORT between countries and regions in 1990-2005. Households in higher socioeconomic groups showed a lower prevalence of morbidity caused by diarrhoea and greater use of ORT in almost all DHS surveys and countries analysed. The pattern was consistent across WHO regions. Analysis of trends did not suggest any significant changes in socioeconomic inequalities in morbidity and treatment of diarrhoea over the study period.

The pattern found was consistent over a large number of surveys. In collaboration with WHO, the DHS programme has developed a standard set of questions regarding diarrhoea that have been tested both within the programme and by WHO [16]. Overall, these questions have been deemed to generate plausible results [17]. There is little reason to believe that they would consistently create a systematic pattern of incorrect answers in different socioeconomic groups. Also, the findings with regard to the prevalence of diarrhoea are in line with expectations, since sanitation and access to water were part of the asset index. Households in higher socioeconomic groups are more likely to have access to good-quality water and sanitation facilities, factors that reduce morbidity associated with diarrhoea [18].

The findings of this study agree with those of earlier studies on socioeconomic disparities in health, which have shown that the health of poorer people is notably worse than that of the wealthier [19-23]. Our findings are also compatible with previous results suggesting that poorer people use health services less frequently, receive inadequate treatment more frequently, and obtain less benefit from primary health care than do wealthier people [19]. In contrast, El Arifeen and collaborators could not document an association between socioeconomic status and childhood illness in a study in Bangladesh. With regard to health care management the study indicated that children with severe illness in the least poor households were three times more likely to seek care from a trained provider than children in the poorest households [24]. Manesh et al. recently found a negative association between mothers' education and morbidity caused by diarrhoea in 44 out of 65 DHS surveys reviewed [25]. However, these authors pointed out that their results were inconsistent with an abundance of previous research and postulated that their findings were due to reporting bias, in that mothers with higher education tend to report more illness in their children. In the present study, we have primarily compared households in the lowest with households in the highest wealth quintiles using an asset index that did not include mothers' educational level. Educational level is an individual-level indicator that might not be the best proxy for household socioeconomic status [26]. Even so, 


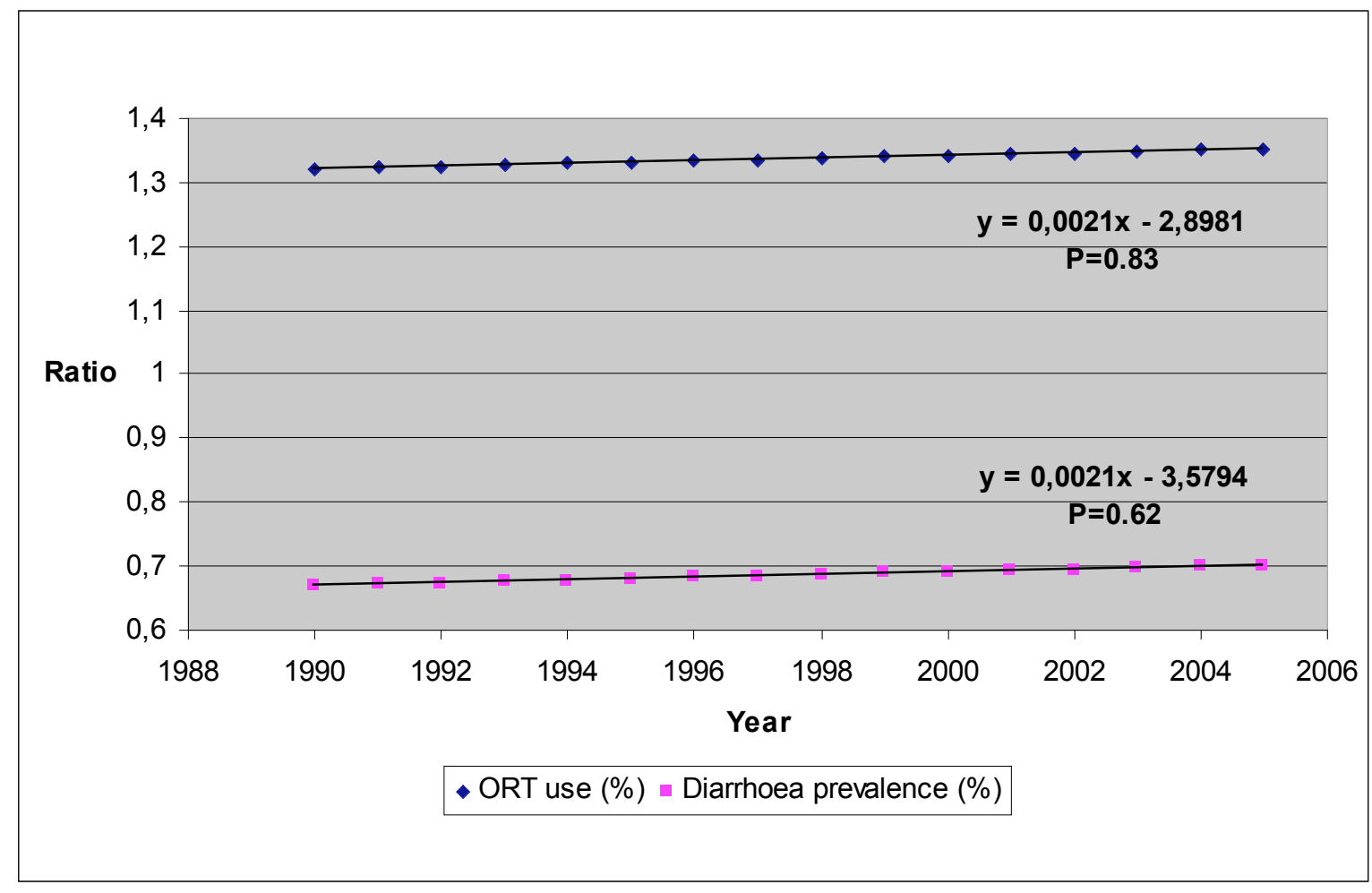

\section{ORT, oral rehydration therapy \\ DHS, Demographic Health Survey}

Fig. (1). Trends in mean rate ratios (rate in highest wealth quintile/rate in lowest wealth quintile) for prevalence of diarrhoea and use of ORT from DHS surveys, 1990-2005.

higher educational level has been found to correlate positively with use of ORT in DHS surveys [27]. Although the possibility of systematic errors, such as reporting errors mentioned by Manesh et al., should be taken into consideration [28], the somewhat contradictory findings in the studies mentioned suggest that the interplay between different indicators of status, such as wealth and mothers' education, needs to be further explored.

The more frequent use of ORT in higher socioeconomic groups in this study points to a potential to increase use of ORT in less wealthy groups. Some of the factors that may account for the difference in use between the richer and the poorer are that the more wealthy have better access to ORT, greater awareness of the importance of appropriate rehydration therapy, more frequent contact with health services where ORT may be recommended or given to the sick child, and more money to buy ORS. Mulholland et al. pointed out in a recent discussion that strategies to improve child survival may reinforce health inequities as they tend to introduce interventions into communities that already have access to basic health care and preventive services, thereby increasing the relative disadvantage experienced by those children without such access [29]. It is possible that this is a factor behind the socioeconomic disparities in use of ORT documented in this study.

Options for reaching the poorest socioeconomic groups in child health programmes have been extensively presented and discussed by Wagstaff et al. [19] and more recently by Taylor $\&$ Marandi [30]. The latter point to the significance of equityoriented health-service delivery, while emphasizing that im- portant actions for improving the health of the poor should be effected outside the conventional health sector.

The present study further documents the importance of targeting the poor in child health-care delivery. Annually, the number of childhood episodes of diarrhoea receiving treatment with ORT could be increased by up to 481 million in the countries surveyed if all socioeconomic groups had adopted the pattern of use of ORT of the best-performing quintile. This potential improvement is of great relevance to earlier findings that too few child diarrhoea episodes are treated with ORT [8] and it motivates further exploration of the reasons behind current differences in use of ORT between socioeconomic groups.

Higher priority should be given to control of diarrhoeal diseases among the poor in low- and middle-income countries. Recommendations on use of oral rehydration therapy in diarrhoea are now mostly integrated in ordinary health services, in many countries within the treatment algorithm for the Integrated Management of Childhood Illnesses (IMCI) [31]. We feel that further attention needs to be given to the promotion of use of oral rehydration therapy both within health services but maybe more important in the community. This is essential in order to attain the Millennium Development Goal of reduction of mortality by two thirds in children aged less than 5 years by 2015. Among the poorest populations, preventive measures should be accelerated, access to ORS/ORT should be increased, rehydration products should made more affordable and information on the importance of using ORT should be disseminated. 


\section{AUTHORS' CONTRIBUTIONS}

Forsberg BC: Lead researcher and author. Conceived study and its design together with Gwatkin and participated fully in all stages of its completion. Drafted first version of article and revised it continuously based on co-authors' comments. Finalized article.

Gwatkin D: Conceived study together with Forsberg. Contributed substantially to study, analysis and interpretation of data, read and commented on drafts and approved the final version. Provided the data on the composite index.

Tomson G: Contributed to the analysis and interpretation of the data, revision of content, and approved of the final version.

Allebeck P: Contributed to the analysis and interpretation of the data, revision of content, and approved of the final version.

Petzold MG: Contributed substantially to conception and design of the study, analysis and interpretation of the data, revision of content, and approval of the final version. Performed statistical analysis.

\section{ROLE OF FUNDING SOURCE}

The research has been carried out within regular work of the researchers at Karolinska Institutet, Stockholm, The World Bank, Washington, and The Nordic School of Public Health, Gothenburg.

\section{ETHICAL CONSIDERATION}

In our work we have adhered to the established Public Health Code of Ethics. The study is based on secondary data and has not involved human participants in the research. As such it does not require approval from an ethical committee.

\section{REFERENCES}

[1] Kosek M, Bern C, Guerrant RL. The global burden of diarrhoeal disease, as estimated from studies published between 1992 and 2000. Bull World Health Organ 2003; 81: 197-204.

[2] Bern C, Martines J, de Zoysa I, Glass RI. The magnitude of the global problem of diarrhoeal disease: a ten-year update. Bull World Health Organ 1992; 70: 705-14.

[3] Black RE, Morris SS, Bryce J. Where and why are 10 million children dying every year? Lancet 2003; 361: 2226-34.

[4] Merson MH. The WHO diarrhoeal diseases control program: the practical application of oral rehydration therapy. in: international conference on oral rehydration therapy (ICORT), Washington: USAID 1983.

[5] WHO. A review of the programme's key indicators and targets. Technical advisory group 14th meeting. Report No. CDD/TAG/93.10. Geneva:WHO 1993.

[6] Fontaine O, Garner P, Bhan MK. Oral rehydration therapy: the simple solution for saving lives. BMJ 2007; 334(Suppl 1): s14.

[7] Victora CG, Bryce J, Fontaine O, Monasch R. Reducing deaths from diarrhoea through oral rehydration therapy. Bull World Health Organ 2000; 78: 1246-55.

[8] Forsberg BC, Petzold MG, Tomson G, Allebeck P. Diarrhoea case management in low- and middle-income countries-an unfinished agenda. Bull World Health Organ 2007; 85: 42-8.
[9] Victora CG, Wagstaff A, Schellenberg JA, Gwatkin D, Claeson M, Habicht JP. Applying an equity lens to child health and mortality: more of the same is not enough. Lancet 2003; 362: 233-41.

[10] Larson CP, Saha UR, Islam R, Roy N. Childhood diarrhoea management practices in Bangladesh: private sector dominance and continued inequities in care. Int J Epidemiol 2006; 35: 1430-9.

[11] Victora CG, Adam T, Bryce J, Smith P. Integrated management of the sick child. In: Jamison D, Breman JG, Measham R, et al, Eds. Disease Control Priorities in Developing Countries, $2^{\text {nd }}$ ed. Oxford: Oxford University Press 2006.

[12] Demographic and Health Surveys. Measure DHS. Calverton: Institute for Resources Development/Macro Systems Inc.; 2007. [cited Dec 12 2007 ]; Available from: www.measuredhs.com

[13] Gwatkin DR, Rutstein SO, Johnson K, Suliman E, Wagstaff A, Amouzou A. Socio-economic differences in health, nutrition and population. Nigeria. Washington: HNP. World Bank 2007.

[14] US Census Bureau. International Data Base (IDB). Washington D.C.: US Census Bureau; 2007. [cited June 10 2007]; Available from: http://www.census.gov/ipc/www/idbnew.html

[15] WHO. Manual for the planning and evaluation of national diarrhoeal diseases control programmes. Geneva: World Health Organization 1984.

[16] WHO. CDD household survey manual: diarrhoea case management, morbidity, and mortality. Geneva: World Health Organization; 1989.

[17] Boerma JT, Black RE, Sommerfelt AE, Rutstein SO, Bicego GT. Accuracy and completeness of mothers' recall of diarrhoea occurrence in pre-school children in demographic and health surveys. Int J Epidemiol 1991; 20: 1073-80.

[18] Cairncross S, Valdmanis V. Water supply, sanitation, and hygiene promotion. In: Jamison D, Breman JG, Measham R, et al, Eds.. Disease Control Priorities in Developing Countries, $2^{\text {nd }}$ ed. Oxford: Oxford University Press 2006.

[19] Wagstaff A, Bustreo F, Bryce J, Claeson M. Child health: reaching the poor. Am J Public Health 2004; 94: 726-36.

[20] Gwatkin DR, Rutstein SO, Johnson K, Suliman E, Wagstaff A, Amouzou A. Socio-economic differences in health, nutrition and population within developing countries. Washington: HNP, World Bank 2007.

[21] Gwatkin DR. Health inequalities and the health of the poor: what do we know? What can we do? Bull World Health Organ 2000; 78: 3-18.

[22] Wagstaff A. Socioeconomic inequalities in child mortality: comparisons across nine developing countries. Bull World Health Organ 2000; 78: 19-29.

[23] Pritchett L, Summers LH. Wealthier is healthier. J Hum Resour 1996; 31: 841-68.

[24] El Arifeen S, Baqui AH, Victora CG, et al. Sex and socioeconomic differentials in child health in rural Bangladesh: findings from a baseline survey for evaluating integrated management of childhood illness. J Health Popul Nutr 2008; 26: 22-35.

[25] Manesh AO, Sheldon TA, Pickett KE, Carr-Hill R. Accuracy of child morbidity data in demographic and health surveys. Int J Epidemiol 2008; 37: 194-200.

[26] Eriksson R. Social class of men, women and families. Sociology 1984; 18: 500-14.

[27] Stallings R. Child morbidity and treatment patterns. Calverton: Institute for Resources Development/Macro Systems Inc 2004.

[28] Forsberg B, van Ginneken J, Nagelkerke N. Cross-sectional household surveys of diarrhoeal diseases - a comparison of data from CDD and DHS programmes. Int J Epidemiol 1993; 22: 1137-45.

[29] Mulholland EK, Smith L, Carneiro I, Becher H, Lehmann D. Equity and child-survival strategies. Bull World Health Organ 2008; 86: 399407.

[30] Taylor S, Marandi A. Social determinants of health and the design of health programmes for the poor. BMJ 2008; 337: 266-9.

[31] Integrated Management of Childhood Illness. A WHO/UNICEF Initiative. Bull World Helath Organ 1997; 75: 5-128. 